\title{
Probing the post-AGB nature of HD 179821^
}

\section{A new radio study of its circumstellar envelope}

\author{
E. Josselin ${ }^{1,2}$ and A. Lèbre ${ }^{2}$ \\ 1 Observatorio Astronómico Nacional (OAN), IGN, Apartado 1143, 28800 Alcalá de Henares, Spain \\ 2 GRAAL-CC72, UMR 5024-ISTEEM, CNRS/Univ. Montpellier II, Place E. Bataillon, 34095 Montpellier Cedex, \\ France \\ Received 25 April 2000 / Accepted 13 November 2000

\begin{abstract}
In spite of recent detailed studies, the nature of HD 179821 is still puzzling. It is classified either as an intermediate-mass post-AGB star or as a massive supergiant. We present here new high sensitivity radio observations of HD 179821 in the circumstellar ${ }^{12} \mathrm{CO}$ and ${ }^{13} \mathrm{CO}$ lines. We also report the new detection of $\mathrm{HCO}^{+}$ in this object. The ${ }^{12} \mathrm{CO}$ line intensities allow estimations of the mass-loss rate of HD 179821 and of the dynamical age of its circumstellar envelope. The comparison between ${ }^{12} \mathrm{CO}$ and ${ }^{13} \mathrm{CO}$ lines indicates an isotopic ratio for carbon of at most 5. Such a low value is characteristic of (post-)AGB stars with low-mass progenitors. Furthermore, the $\mathrm{HCO}^{+}$molecular ion indicates that an active photochemistry occurs in the envelope of HD 179821, probably induced by UV photons emitted as the central post-AGB star warms up and/or by shocks generated by interacting winds.
\end{abstract}

Key words. stars: AGB and post-AGB - stars: circumstellar matter - stars: individual: HD 179821

\section{Introduction}

The post-Main Sequence evolution of stars is strongly dependent on their initial mass. On the one hand, low- and intermediate-mass stars (initial mass $M_{\mathrm{i}} \lesssim 8 M_{\odot}$ ) evolve along the Red Giant Branch (RGB) and Asymptotic Giant Branch (AGB) before becoming Planetary Nebulae (PNe) (Habing 1996). The transition phase between AGB stars and $\mathrm{PNe}$, designed as the post-AGB phase or proto-PNe (PPNe) phase, is very brief $\left(\sim 10^{3}\right.$ yrs, Blöcker 1995), but its study is a clue to understand the shaping of PNe and the properties of their central stars (Kwok 1993). On the other hand, massive stars $\left(M_{\mathrm{i}} \gtrsim 8-10 M_{\odot}\right)$ evolve to the Red Supergiant phase, where they eventually experiment a blue loop before reaching the Wolf-Rayet stage and/or explode as a supernova (Langer 1998). For very massive stars $\left(M_{\mathrm{i}} \gtrsim 40 M_{\odot}\right)$ this blue loop occurs at earlier spectral types, because of a stability limit (de Jager 1991). In both evolutionary scenarios, the key phenomenon is mass loss, which generates a circumstellar envelope ( $\mathrm{CsE}$ ).

The distinction between post-AGB stars and supergiants is a difficult task. Both types of objects are of luminosity class I and they undergo low-amplitude variations (Kwok 1993). Their circumstellar envelopes can present large expansion velocities $\left(V_{\exp } \gtrsim 15 \mathrm{~km} \mathrm{~s}^{-1}\right)$. While so-

Send offprint requests to: E. Josselin,

e-mail: josselin@graal.univ-montp2.fr

* Based on observations carried out with the IRAM $30 \mathrm{~m}$ telescope. called "high latitude supergiants" are now widely believed to be post-AGB stars (van der Veen et al. 1993), the nature of low-latitude stars of spectral types B-G and luminosity class I is more difficult to assess. This problem is generally addressed through high-resolution optical spectroscopy, but even then, definite conclusions are difficult to draw.

HD $179821(=$ SAO $124414=$ IRAS 19114+0002) is one of these puzzling objects, being classified either as a post-AGB star $\left(M_{\mathrm{i}} \sim 3-4 M_{\odot}\right.$; e.g. Reddy \& Hrivnak $1999)$ or as a massive supergiant $\left(M_{\mathrm{i}} \sim 30 M_{\odot}\right.$; e.g. Jura \& Werner 1999). We present here an analysis of new millimeter observations of this source, which clearly favour the post-AGB hypothesis.

A summary of the properties of HD 179821 is given in Sect. 2, with particular emphasis on the controversy about whether it is a post-AGB star or a massive supergiant. A discussion about its distance is also given in Sect. 2. The radio observations are presented and discussed in Sect. 3, in the scope of bringing new constraints on the nature HD 179821.

\section{Source properties}

HD 179821 is of spectral type G5 Ia (Hrivnak et al. 1989) and is located at a rather low galactic latitude ( $b \mathrm{II}=$ $\left.-4.96^{\circ}\right)$. The detection of $\mathrm{OH}$ maser lines implies that it is an oxygen-rich object (Likkel 1989). It has a doublepeaked spectral energy distribution (SED), with equal 
amounts of energy from the photosphere and from the cold dust circumstellar shell (van der Veen et al. 1989). This SED is similar to other post-AGB stars which have experienced interruption of mass loss (Kwok 1993). This is likely related to the detached dust shell observed in the mid-infrared (Jura \& Werner 1999). Other remarkable parameters of this object are its expansion and macroturbulence velocities: $V_{\exp } \sim 35 \mathrm{~km} \mathrm{~s}^{-1}$ (Bujarrabal et al. 1992) and $V_{\text {macroturb }} \sim 20 \mathrm{~km} \mathrm{~s}^{-1}$ (Thévenin et al. 2000). These values are relatively high compared to similar objects and, as will be discussed later, affect both the dynamics and the abundance determinations.

\subsection{Controversy about the nature of HD 179821}

Reddy \& Hrivnak (1999) made a detailed analysis of highresolution optical spectra of this source. Their LTE analysis gives an effective temperature $\left(T_{\text {eff }}\right)$ of $6750 \mathrm{~K}$, suggesting a spectral type $\mathrm{F}$, in contradiction with a previous analysis (Hrivnak et al. 1989). The high $s$-process elements abundances support the idea that HD 179821 is a postAGB object. However, interstellar absorption components in the NaI doublet suggest a large distance for HD 179821 ( $\gtrsim 4 \mathrm{kpc}$ ), implying a high luminosity $\left(M_{\mathrm{bol}} \sim-9 \mathrm{mag}\right)$ and a massive supergiant nature $\left(15-20 M_{\odot}\right)$.

On the contrary, Thévenin et al. (2000) have based their composition analysis on medium-resolution spectra, arguing that the strong macroturbulence in HD 179821 dominates the spectral line widths and implies strong line blending. They rejected a high value for $T_{\text {eff }}$ and they adopted a value of $5660 \mathrm{~K}$, corresponding to a spectral type G0, more consistent with the usual spectral classification. They did not find the high $s$-process elements abundances and they pointed out that moderate metal deficiency is inconsistent with the massive population I supergiant hypothesis.

Based on mid-infrared observations, Jura \& Werner (1999) studied the detached dust shell around this star. All their results were interpreted assuming a $30 M_{\odot}$ supergiant, as they argued that the high LSR $\left(99 \mathrm{~km} \mathrm{~s}^{-1}\right)$ and outflow $\left(35 \mathrm{~km} \mathrm{~s}^{-1}\right.$ ) velocities (Zuckerman \& Dyck 1986; Bujarrabal et al. 1992) favour this hypothesis. Nevertheless, such high expansion velocities have already been observed in well-identified post-AGB stars (e.g. OH 231.8+4.2 with $V_{\text {exp }}=30 \mathrm{~km} \mathrm{~s}^{-1}$; Omont et al. 1993). Another apparently strong argument in favour of a massive supergiant concerns the ultraviolet dust opacity, the parameter they denote $\chi_{\mathrm{UV}}$. Based on the model of Jura (1996) and assuming a distance of $1 \mathrm{kpc}$, they find $\chi_{\mathrm{UV}}=$ $410^{5} \mathrm{~cm}^{2} \mathrm{~g}^{-1}$, well above the values found for other AGB stars (by a factor $\sim 2.7$ ). However, the determination of $\chi_{\mathrm{UV}}$ contains some uncertainties. Indeed, according to Eq. (2) in Jura (1996), one finds $\chi_{\mathrm{UV}}=\sigma_{\mathrm{gr}} / m_{\mathrm{gr}}$, where $\sigma_{\mathrm{gr}}$ is the average grain cross section and $m_{\mathrm{gr}}$ is the grain mass, and so $\chi_{\mathrm{UV}} \propto a^{-1}$, with $a$ being the grain size. Now, only upper limits on the grain size $a$ are given by Jura (1996) for AGB stars, so that the resulting estimations of $\chi_{\mathrm{UV}}$ are lower limits. The estimation for HD 179821 may then be consistent with those for other low-mass stars. Finally, Jura \& Werner (1999) derived a total mass-loss rate for HD 179821 of $\sim 410^{-4} M_{\odot} \mathrm{yr}^{-1}$ which occured about 1800 yrs ago, for a distance of $6 \mathrm{kpc}$ and a gas-to-dust ratio of 100 . They also found that this rate was approximately constant with time but probably not isotropic.

\subsection{The problem of distance}

One of the main issues in determining the nature of HD 179821 is the determination of its distance. The kinematic distance of $6 \mathrm{kpc}$ (Zuckerman \& Dyck 1986) is widely used to favour the massive star hypothesis. One must keep in mind however, that this value is very uncertain.

First of all, using a simple approximation relation for the velocity structure of the galactic disk (Lang 1980), the distance is undetermined for a galactic longitude $l \mathrm{II}=$ $35.6^{\circ}$ and $V_{\mathrm{lsr}} \geq 95 \mathrm{~km} \mathrm{~s}^{-1}$. At this longitude, a distance of $6 \mathrm{kpc}$ is obtained for $V_{\mathrm{lsr}}=90 \mathrm{~km} \mathrm{~s}^{-1}$.

Secondly, as emphasized by Kastner et al. (1993), this method is valid only if the star lies within the galactic disk. For a distance of $6 \mathrm{kpc}$, the height of HD 179821 above the galactic plane would be 524 pc, which is relatively high, and well above the scale height for $30 M_{\odot}$ stars ( $\sim 90$ pc, Miller \& Scalo 1979). A height of 90 pc would correspond to a distance of about $1 \mathrm{kpc}$, but the resulting low luminosity $\left(\sim 10^{4} L_{\odot}\right)$ is then in contradiction with the hypothesis of a massive supergiant. Then, the high radial velocity may rather reflect a past runaway phenomenon (Kastner et al. 1993).

The hypothesis that HD 179821 is a low-mass postAGB star thus seems to be more robust. We consistently adopt a distance of $1 \mathrm{kpc}$ for HD 179821 in the analysis of the observations we present in the next section, keeping in mind that it may be uncertain by about $50 \%$ (Bujarrabal et al. 1992). This results in an uncertainty of about a factor 2 for the derived mass-loss rate, and more generally for all parameters varying as the square of the distance. We will show that, within these uncertainties, this distance is fully consistent with the properties of its circumstellar envelope.

\section{The molecular envelope of HD 179821}

\subsection{Previous radio observations}

Millimeter observations of molecular lines were reported by Bujarrabal et al. (1992). They detected the ${ }^{12} \mathrm{CO}$ and ${ }^{13} \mathrm{CO}(1-0)$ and $(2-1)$ lines, as well as $\mathrm{SiO}(2-1)$ and $(3-2)$ lines. They did not detect the $\mathrm{CS}(3-2)$ and the $\mathrm{HCN}(1-$ $0)$ lines, which confirms the O-rich nature of HD 179821. The $\mathrm{HCO}^{+}(1-0)$ line was not detected with a rms of $0.1 \mathrm{~K}$ (main beam temperature at the IRAM $30 \mathrm{~m}$ telescope). Bujarrabal et al. (1992) also found clear evidence for departures from spherical symmetry of the circumstellar 
envelope. Adopting a mass-loss rate of $10^{-3} M_{\odot} \mathrm{yr}^{-1}$ for a distance of $1.5 \mathrm{kpc}$ (Hrivnak et al. 1989), they found a slight deficiency of ${ }^{12} \mathrm{CO}\left({ }^{12} \mathrm{CO} / \mathrm{H}_{2}=10^{-4}\right)$, compared to the usual value believed to be present in O-rich AGB stars.

Omont et al. (1993) have also reported the detection of the $\mathrm{H}_{2} \mathrm{~S}\left(1_{1,0}-1_{0,1}\right)$ line at a $3 \sigma$ level. Bujarrabal et al. (1994) detected $\mathrm{HNC}(1-0)$ and tentatively $\mathrm{SO}\left(6_{5}-\right.$ $\left.5_{4}\right)$. These two latter molecules may be a sign of an active photochemistry and/or the presence of shocks in the envelope.

\subsection{New radio observations}

Regarding the recent progress in sensitivity and calibration of millimeter detectors, we performed in August 1999 new observations at the IRAM $30 \mathrm{~m}$ telescope, on Pico Veleta (near Granada, Spain). Four lines were observed simultaneously: ${ }^{12} \mathrm{CO}(1-0)$ and $(2-1),{ }^{13} \mathrm{CO}(2-1)$ and $\mathrm{HCO}^{+}(1-0)$. We used SIS receivers at 3 and $1 \mathrm{~mm}$. The half-power beam width is $22^{\prime \prime}$ and $10^{\prime \prime}$ at 3 and $1 \mathrm{~mm}$ respectively. The spectrometers were $256 \times 1 \mathrm{MHz}$ filter banks. The observations were done by wobbling the secondary mirror to a distance of $2^{\prime}$ from the source. This provides very flat baselines and thus ensures precise calibration. The pointing accuracy, checked every hour, was within $3^{\prime \prime}$. Calibration was achieved with a chopper wheel. The intensities given hereafter are in units of main beam brightness temperature.

As HD 179821 presents an extended envelope, we made small maps covering the emission area, with offsets ranging from $-20^{\prime \prime}$ to $20^{\prime \prime}$, with step of $10^{\prime \prime}$ in both right ascension and declination. These maps are thus fully sampled at $3 \mathrm{~mm}\left({ }^{12} \mathrm{CO}(1-0)\right.$ and $\mathrm{HCO}^{+}(1-0)$ lines $)$. The parameters of the observed lines are given in Table 1 and spectra are displayed in Fig. 1. For the CO lines the given parameters and the displayed spectra correspond to the central positions. For the $\mathrm{HCO}^{+}(1-0)$ line, both parameters and spectrum correspond to the average over nine positions, with position offsets ranging from $-10^{\prime \prime}$ to $10^{\prime \prime}$ in both directions. This was done to increase the signal-to-noise ratio for this very faint line. This allowed the detection of this line at a $4 \sigma$ level. Although it is weak, this detection is reliable as it has the same kinematic properties (radial and expansion velocities) as the strong CO lines.

\subsection{Results}

The emission in all CO lines is resolved. We obtain similar extensions for ${ }^{12} \mathrm{CO}(2-1)$ and ${ }^{13} \mathrm{CO}(2-1)$, of about $14^{\prime \prime}$, which implies a deconvolved radius of $4.9^{\prime \prime}$. This extension is compatible with the one already found by Bujarrabal et al. (1992), i.e. $18^{\prime \prime} \times 14^{\prime \prime}$, as such asymmetry is not detectable with our sampling.

The ${ }^{12} \mathrm{CO}$ lines are globally parabolic, suggesting optically thick emission. Such optical thickness is also indicated by the observed ${ }^{12} \mathrm{CO}(2-1) /{ }^{12} \mathrm{CO}(1-0)$ line ratio $(\sim 2.9)$. Because of beam dilution effects, the intrin-
Table 1. Results of the observations. The values correspond to the central position of the CsE, except for the $\mathrm{HCO}^{+}(1-0)$ line where the values correspond to the average over the CsE

\begin{tabular}{cccccc}
\hline line & $\begin{array}{c}V_{\text {lsr }} \\
\left(\mathrm{km} \mathrm{s}^{-1}\right)\end{array}$ & $\begin{array}{c}V_{\text {exp }} \\
\left(\mathrm{K} \mathrm{km} \mathrm{s}^{-1}\right)\end{array}$ & $\begin{array}{c}T_{\mathrm{mb}} \\
(\mathrm{K})\end{array}$ & $\begin{array}{c}\mathrm{rms} \\
(\mathrm{K})\end{array}$ \\
\hline${ }^{12} \mathrm{CO}(1-0)$ & 96 & 36 & 64.5 & 1.17 & 0.02 \\
${ }^{12} \mathrm{CO}(2-1)$ & 97 & 37 & 172.4 & 3.45 & 0.08 \\
${ }^{13} \mathrm{CO}(2-1)$ & 98 & 34 & 53.8 & 1.24 & 0.09 \\
$\mathrm{HCO}^{+}(1-0)$ & 95 & 35 & 0.3 & 0.01 & 0.003 \\
\hline
\end{tabular}

sic value of this ratio is close to 1 . One should note that supergiants generally exhibit larger values for this ratio ( $\gtrsim 4$ ), probably because of different excitation processes around massive stars (Josselin et al. 1998). Furthermore, all the CO lines are clearly asymmetric, with sharper profiles on the red edge of the lines. This may be linked to the anisotropy of the mass loss, pointed out by Jura \& Werner (1999). They suggest that the observed asymmetry in the mid-infrared can be explained by different expansion velocities towards the north and the south. High resolution maps of the $\mathrm{CO}$ emission would be necessary to see where the relative excess of blue emission comes from and eventually confirm this explanation.

The ${ }^{13} \mathrm{CO}(2-1)$ line should be optically thinner. This explains the more complex line shape, where features appear, in particular on the red side of the line. This has already been observed by Bujarrabal et al. (1992), who suggest that these features are due to components of receding gas in addition to the "standard" flow.

\section{Analysis}

\subsection{Dynamics of the envelope}

The deconvolved extent of the envelope corresponds to a linear radius of the molecular envelope, $R_{\mathrm{co}}=710^{16} \mathrm{~cm}$ for a distance of $1 \mathrm{kpc}$. For an expansion velocity of $\sim 35 \mathrm{~km} \mathrm{~s}^{-1}$, this implies a kinematic age $t_{\text {kin }}=570 \mathrm{yrs}$.

After correction for distance, this is half the age obtained from the size of the dust envelope (Kastner \& Weintraub 1995). This difference may result from photodissociation of $\mathrm{CO}$ by interstellar UV radiation and/or condensation onto grains. Furthermore, as mid-infrared dust emission and millimeter CO lines trace a different epoch of mass loss, this discrepancy between age estimations may be attributed to time variations of the mass-loss rate (Delfosse et al. 1997 and references therein).

For an optically thick $\mathrm{CO}(1-0)$ line the mass-loss rate is estimated from the formula given by Loup et al. (1993):

$\dot{M}=2.710^{-11} \frac{T_{\mathrm{mb}} V_{\mathrm{exp}}^{2} D^{2}}{f_{\mathrm{co}}^{0.85} F\left(R_{\mathrm{co}}\right)}$

where $T_{\mathrm{mb}}$ is the main beam temperature of the $\mathrm{CO}(1-$ $0)$ transition measured by a $30 \mathrm{~m}$ antenna, $f_{\text {co }}$ is the CO abundance relative to $\mathrm{H}_{2}$ and $F\left(R_{\mathrm{co}}\right)$ is a function of the 

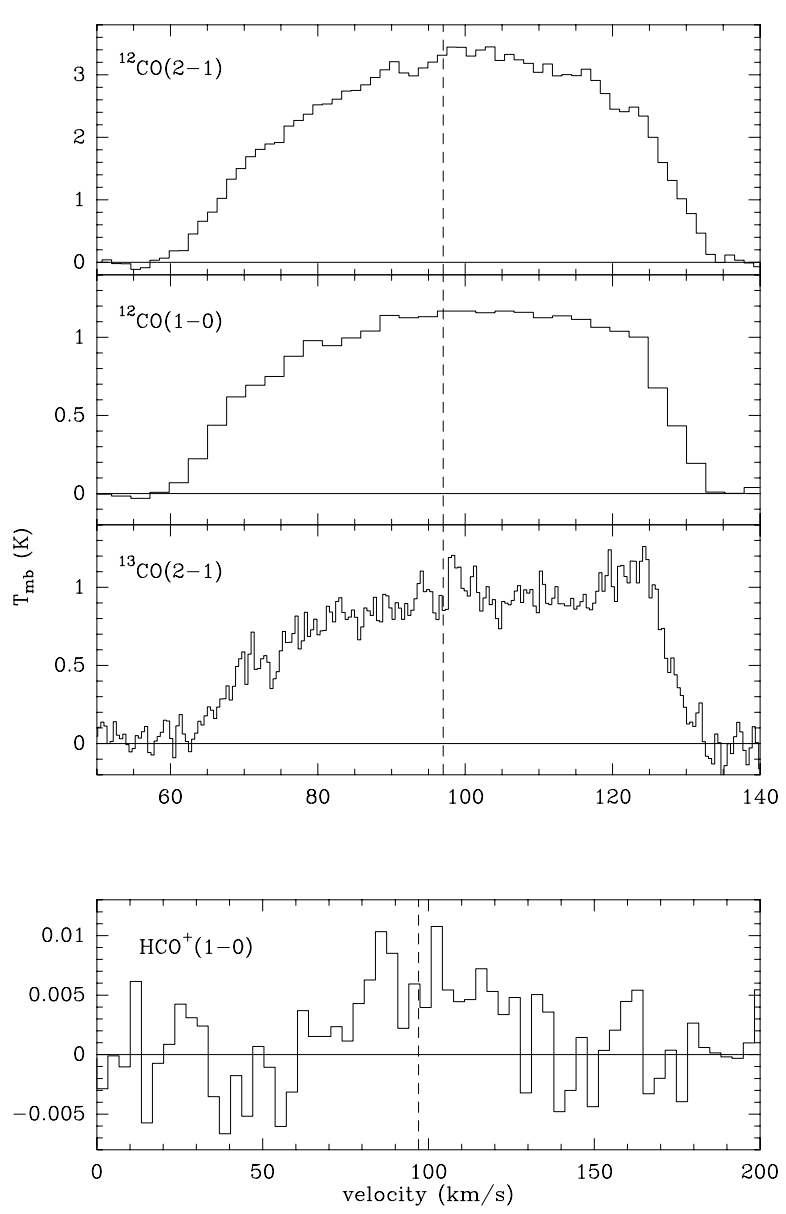

Fig. 1. Spectra of the observed lines. The vertical dashed line indicates the LSR velocity deduced from the ${ }^{12} \mathrm{CO}(2-1)$ line. The CO lines are zoomed along the velocity range where emission is found in order to emphasize the structure of these lines. The $\mathrm{HCO}^{+}(1-0)$ line is shown on a wider velocity range so as to show the reliability of its detection

radius of the $\mathrm{CO}$ envelope which accounts for photodissociation by interstellar UV radiation (Fig. 2 in Loup et al. 1993). A typical value for $f_{\text {co }}$ is $510^{-4}$ for an oxygen-rich medium, assuming that all $\mathrm{C}$ is in form of $\mathrm{CO}$ within the photodissociation radius. For a distance of $1 \mathrm{kpc}$ we obtain

$\dot{M}=210^{-4} M_{\odot} \mathrm{yr}^{-1}$.

This value is in good agreement with the mass loss rates inferred for post-AGB stars (Bujarrabal 1999) but it is much lower than the value of $10^{-3} M_{\odot} \mathrm{yr}^{-1}$ adopted by Bujarrabal et al. (1992). The mass of the molecular envelope, and thus the mass-loss rate, varies as the square of the linear size of the envelope. Assuming a distance of $1.5 \mathrm{kpc}$ as they did and taking the largest angular size they observed $\left(18^{\prime \prime}\right)$, the linear size becomes $1.610^{17} \mathrm{~cm}$, i.e. a factor 2.3 larger than that obtained for an angular size of $14^{\prime \prime}$ at $1 \mathrm{kpc}$. The resulting mass-loss rate will thus be a factor $\sim 5$ larger, which is necessary to reconcile both estimations. Our estimation is also higher than that of Jura \& Werner (1999), after correction for the distance. Here again, this must reveal strong time variations of the mass-loss rate.
Assuming a stellar luminosity of $\sim 10^{4} L_{\odot}$ at $1 \mathrm{kpc}$ (based on the bolometric magnitude found by integrating the spectral energy distribution; see Bujarrabal et al. 1992 for a detailed discussion of the determination of the luminosity) and the mass-loss rate we propose in the present work, the ratio between the momentum of the envelope $\left(\dot{M} V_{\text {exp }}\right)$ and the stellar momentum $\left(L_{\star} / c\right)$ is $\sim 34$. This value is inconsistent with models of radiation-driven mass loss, but it is still consistent with the idea of a quasiexplosive ejection of matter during the post-AGB phase (Bujarrabal 1999).

\subsection{The ${ }^{12} \mathrm{C} /{ }^{13} \mathrm{C}$ isotopic ratio}

The observed intensity ratio $I\left({ }^{12} \mathrm{CO}(2-1)\right) / I\left({ }^{13} \mathrm{CO}(2-1)\right)$ is 3.2 . However, this value may not give directly the ${ }^{12} \mathrm{C} /{ }^{13} \mathrm{C}$ isotopic ratio.

First, we assume that the filling factor is the same for both lines, observed with the same beam size. It is also reasonable to assume that the rotational levels are thermalized, since the dipole moment of CO is quite small ( $\sim 0.1$ Debye). Then, the main effect which has to be taken into account is the ${ }^{12} \mathrm{CO}(2-1)$ line saturation, due to optical thickness. Other possible effects are selective photodissociation $-{ }^{13} \mathrm{CO}$ being more easily photodissociated - and chemical fractionation through the reaction:

${ }^{12} \mathrm{CO}+{ }^{13} \mathrm{C}^{+} \rightarrow{ }^{13} \mathrm{CO}+{ }^{12} \mathrm{C}^{+}$.

These two latter effects are generally thought to compensate each other (see Mamon et al. 1988 for a detailed discussion).

Saturation effects should not be higher than $30 \%$ (Knapp \& Morris 1985). We thus obtain an upper limit of $\sim 5$ for the ${ }^{12} \mathrm{C} /{ }^{13} \mathrm{C}$ abundance ratio. More accurate determination would require radiative transfer calculations, but also ingredients such as the temperature profile which is poorly constrained.

This value is remarkably low, but not unusual for a low-mass post-AGB star. A typical value for RGB stars of initial mass $2-4 M_{\odot}$ is $\sim 20$. This value can drop down to $\lesssim 10$ for a $1 M_{\odot}$ star, in the case of extra-mixing processes (Charbonnel 1995) and can decrease during evolution from the RGB to the AGB, even when third dredge-up occurs (Wasserburg et al. 1995). On the contrary, these extramixing processes such as cool bottom burning should not occur in more massive stars. Finally, additional processes should be taken into account to reproduce the low values observed in the latest stages of stellar evolution (see Palla et al. 2000 for a more detailed discussion). Indeed, for PNe of initial mass $\sim 1-2.5 M_{\odot}$, Palla et al. (2000) found an isotopic ratio similar to that of HD 179821. Interestingly, for such a low initial mass, the star probably did not experience a 3rd dredge-up (Mowlawi 1999 and references therein), as precisely found by Thévenin et al. (2000). 


\subsection{Circumstellar chemistry}

Among the molecules detected in the circumstellar envelope of HD 179821, the most remarkable ones are HNC, SO (Bujarrabal et al. 1994) and $\mathrm{HCO}^{+}$(our present work). Indeed, these molecules may result from an active photochemistry, generated by UV photons emitted by the central star as it warms up, or can be produced in shocks. Shocks may occur around post-AGB stars, in particular in the interacting winds scenario.

$\mathrm{HCO}^{+}$may be formed through two paths. The first one invokes the reaction of $\mathrm{H}_{3}^{+}$with $\mathrm{CO}$, but the survival of the $\mathrm{H}_{3}^{+}$ion is very short, because of very effective dissociative recombination (Bachiller et al. 1997). Thus, $\mathrm{HCO}^{+}$ is probably essentially formed through the reaction

$\mathrm{CO}^{+}+\mathrm{H}_{2} \rightarrow \mathrm{HCO}^{+}+\mathrm{H}$

and is likely to be destroyed by photodissociation, back to $\mathrm{CO}^{+}$, or dissociative recombination, forming $\mathrm{CO}$ (Mamon et al. 1987). Then, $\mathrm{HCO}^{+}$is associated with $\mathrm{CO}^{+}$, which should be formed through the propagation of an ionization front into neutral gas rich in CO (Howe \& Williams 1998 and references therein). The initiation of such ionization front is one of the characteristics of PPNe (Kwok 1993) and $\mathrm{HCO}^{+}$seems indeed to be formed during this stage (Bachiller et al. 1997).

An ionization front may also be formed by UV radiation from the chromospheres of massive supergiants. But up to now, to our knowledge, $\mathrm{HCO}^{+}$has never been detected in the envelope of a supergiant. Thus, its detection seems to indicate that the star is of low or intermediate initial mass.

\section{Conclusion}

The nature of HD 179821 is subject to debate. It is either a massive supergiant or a rather low mass post-AGB star.

The most widely used argument in favour of the massive supergiant hypothesis is the kinematic distance, estimated to be about $6 \mathrm{kpc}$. We showed that it is very doubtful, as the method used for this determination is not valid regarding the galactic coordinates of HD 179821 and leads to a height above the galactic plane $(\sim 500 \mathrm{pc})$ too large for a massive star.

The analysis of our new high-sensitivity radio observations favour the hypothesis that HD 179821 is a low-mass post-AGB star. First, the ${ }^{12} \mathrm{C} /{ }^{13} \mathrm{C}$ isotopic ratio is rather low, at most 5. Such a value has been observed towards PPNe and PNe of low initial mass $\left(\sim 2 M_{\odot}\right)$ and can be explained thanks to extra-mixing processes, but it is inconsistent with our current knowledge of mixing processes in massive stars. Furthermore, we detect for the first time the $\mathrm{HCO}^{+}(1-0)$ line from HD 179821, which is a signature of an active photochemistry, a characteristic of post-AGB stars. Our conclusions are also fully consistent with recent determinations of the chemical composition of HD 179821 (Thévenin et al. 2000).

Additional observations, such as spectrophotometric follow-up and abundance determinations from high- resolution spectroscopy over a wider range of wavelengths than those thus far performed, may bring new independent constraints on the nature of HD 179821.

Acknowledgements. We are grateful to R. Bachiller for providing complementary observations which confirmed the validity of our calibration and for fruitful discussions. We also thank our anonymous referee for comments which greatly improved the presentation of our results. E. J. acknowledges partial support from Spanish grants PB 96-104 and SB 97-22043.

\section{References}

Bachiller, R., Forveille, T., Huggins, P. J., \& Cox, P. 1997, A\&A, 324, 1123

Blöcker, T. 1995, A\&A, 299, 755

Bujarrabal, V., Alcolea, J., \& Planesas, P. 1992, A\&A, 257, 701

Bujarrabal, V., Fuente, A., \& Omont, A. 1994, A\&A, 285, 247

Bujarrabal, V. 1999, in Asymptotic Giant Branch Stars, IAU Symp. 191, ed. T. Le Bertre, A. Lèbre, \& C. Waelkens, 363 Charbonnel, C. 1995, ApJ, 453, L41

de Jager, C. 1991, A\&A, 244, 131

Delfosse, X., Kahane, C., \& Forveille, T. 1997, A\&A, 320, 249

Habing, H. J. 1996, A\&AR, 7, 97

Howe, D. A., \& Williams, D. A. 1998, in The molecular astrophysics of stars and galaxies, ed. T. W. Hartquist, \& D. A. Williams (Oxford University Press), 347

Hrivnak, B. J., Kwok, S., \& Volk, K. M. 1989, ApJ, 346, 265

Josselin, E., Loup, C., Omont, A., et al. 1998, A\&AS, 129, 45

Jura, M., \& Werner, M. W. 1999, ApJ, 525, L113

Jura, M. 1996, ApJ, 472, 806

Kastner, J. H., Forveille, T., Zuckerman B., \& Omont, A. 1993, A\&A, 275, 163

Kastner, J. H., \& Weintraub, D. A. 1995, ApJ, 452, 833

Knapp, G. R., \& Morris, M. 1985, ApJ, 292, 640

Kwok, S. 1993, ARA\&A, 31, 63

Lang, K. R. 1980, Astrophysical Formulae, 2nd Edition (Springer Verlag), 531

Langer, N. 1998, in New views of the Magellanic Clouds, IAU Symp. 190, in press

Likkel, L. 1989, ApJ, 344, 350

Loup, C., Forveille, T., Omont, A., \& Paul, J. F. 1993, A\&AS, 99, 291

Mamon, G. A., Glassgold, A. E., \& Omont, A. 1987, ApJ, 323, 306

Mamon, G. A., Glassgold, A. E., \& Huggins, P. J. 1988, ApJ, 328, 797

Miller, G. E., \& Scalo, J. M. 1979, ApJS, 41, 513

Mowlawi, N. 1999, in Asymptotic Giant Branch Stars, IAU Symp. 191, ed. T. Le Bertre, A. Lèbre, \& C. Waelkens, 47

Omont, A., Lucas, R., Morris, M., \& Guilloteau, S. 1993, A\&A, 267, 490

Palla, F., Bachiller, R., Stanghellini, L., Tosi, M., \& Galli, D. 2000, A\&A, 355, 69

Reddy, B. E., \& Hrivnak, B. J. 1999, AJ, 117, 1834

Thévenin, F., Parthasarathy, M., \& Jasniewicz, G. 2000, A\&A, 359,138

van der Veen, W. E. C. J., Habing, H. J., \& Geballe, T. R. 1989, A\&A, 226, 108

van der Veen, W. E. C. J., Trams, N. R., \& Waters, L. B. F. M. 1993, A\&A, 269, 231

Wasserburg, G. J., Boothroyd, A. I, \& Sackmann, I. J. 1995, ApJ, 447, L37

Zuckerman, B., \& Dyck, H. M. 1986, ApJ, 311, 345 